\title{
ANDROID APPLICATION FOR S-PARK SYSTEM
}

\author{
Gaurav Kate, Kaustubh Dave', Saprem Kulkarni², R. B. Dhumale ${ }^{3}$ \\ Department of E \& TC Engg., Sinhgad College of Engineering, Pune, India.
}

\begin{abstract}
Finding a parking space in most metropolitan areas, during the rush hours, is difficult for drivers. The problem arises from not knowing where is the empty space; even if it is known, many vehicles may pursue very limited parking spaces to cause serious traffic congestion. In this project, a prototype of smart parking ( $S$-Park) system is designed and implemented that allows drivers to effectively find and reserve the empty parking spaces using an android application connected to Internet. By periodically polling the parking status from the sensor networks deployed in parking lots, the reservation status is affected by the change of physical parking status. The drivers are allowed to access the server on their Android smartphones. The proposed smart parking policy has the potential to solve the problems of parking systems, as well as reduce traffic congestion caused by parking searching thus making the system two-way efficient, that is, cost wise and time wise.
\end{abstract}

Key Words: Smart parking system, Android based application, Embedded server, One time password.

\section{INTRODUCTION}

Searching for the parking space in metropolitan areas is now becoming time consuming. Due to mismanagement in the parking space, it is difficult for drivers to find the parking slot which results more traffic congestion and air pollution by constantly wandering in certain area for available parking space. Many smart parking systems are trying to reduce such traffic congestion and improve convenience for drivers. There are some currently working parking guidance systems which obtain available parking space information from deployed sensors and provide it to drivers[5]. However, these systems are not helpful to guide the drivers to find the proper empty parking slot in the parking space. When available spaces are limited, and if more drivers are heading towards these spaces, it will cause severe congestion. Even though driver reaches the parking area, there are certain problems such as blind search [3] in finding the empty slots. It causes not only wastage of money but wastage of fuel also. To solve the this problem, techniques like buffered parking information sharing(BPIS) [3] are used. But determining the number of buffered spaces is difficult. If buffered spaces are low, there is problem of "multiple-carschase-single-space". If buffer is large then efficient utilizations of parking slots is very low.

There are some systems like smart parking service based on wireless sensor networks [1] which uses wireless sensors to effectively find the available parking space. But to use this system, additional hardware needs to be installed in the car which is not feasible. New Smart Parking System Based on Resource Allocation and Reservations [2] which has facility to reserve the parking slot for fixed amount of time. However, if the driver does not follow the timings as per reservation it causes inconvenience for others.

The solution proposed in this project works as when user wants to park car at the parking, the user uses the Android application to check for available parking slots. The availability is updated by the server when the availability is checked by the user. If the slot is available, the user then enters the payment mode and payment details. These details are stored on the server and an OTP (One Time Password) is generated, which is given to the user after payment is verified. This OTP is then valid for a certain amount of time after which it expires. The user when reaches the parking, is required to enter the valid OTP and based on the current availability of the parking slots, the nearest available parking slot is allocated.

The proposed solution provides an efficient method for providing parking space to the user as they are allotted specific and unique OTP (One Time Password) on successful booking of parking slots. This eliminates the need of human intervention at the parking lot, as the user simply needs to enter the OTP at the console provided at the entrance of the parking lot. Also the provided OTP remains valid for a pre-defined amount of time which eliminates the problem of the user booking the space and not showing up which can result in under utilization of the parking lot.

\section{METHDOLOGY}

In the system, the server periodically sends signals to the sensor controller which checks for the availability using the proximity sensors installed at each of the parking spaces. Based on the statuses of these proximity sensors, the occupancy and availability is updated on the server and this status is then shown to the user whenever availability is checked on the android application. Now, when the user wants to book a parking space, and a space is available, payment method is chosen and payment details are entered by the user. On authentication of the payment details, a space is booked on the server and a One Time Password (OTP) is generated which is a unique identification for the user. That OTP is stored at both places, that is, at the user side (android application) and server side. Now when the user reaches the parking, the OTP is required to be entered on the console.

This entered OTP is then verified with respect to correctness as well as time limit with the server and indicated to the user 
appropriately. On verification, the server checks for the nearest available parking space and allocates it to the user and displays the allotted slot's location on the display. On getting the location, the user parks the car and the corresponding occupancy status is updated on the server using the proximity sensors.

As seen from the Figure 1, the central controlling unit of the system is the server which performs multiple functions such as interfacing with the console, that is, display and keypad, connecting to the sensor controller to update the status of the parking spaces, that is, whether empty or occupied and lastly as a server so as to receive requests from the client side, that is, smartphone connected to the network to check availability as well as book parking spaces. It also checks for the correctness of the entered OTP and gives the corresponding indication. The sensors, with the help of sensor controller, at each of the parking slots check for the occupancy of their parking spaces and give the corresponding status to the server which then updates its database. The smartphone then on the user's side is used to check the availability status of the parking spaces at a particular parking lot and if available book a space. Each slot is provided with ultrasonic sensor with 8-bit MCU( microcontroller unit) and each MCU is connected to the embedded server via one wire protocol having TDMA type of multiplexing [4].

\section{EXPERIMENTATION}

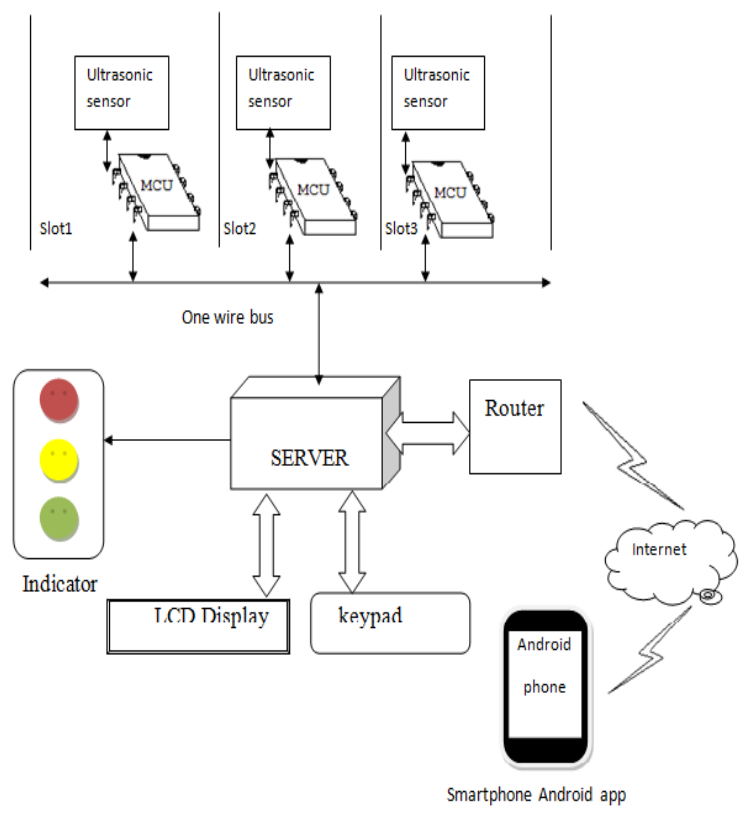

Fig. 1:Block diagram of proposed S-Park system

Ultrasonic sensor (HC-SR 04) is interfaced with the sensor controller as shown in the above Figure 2. The ultrasonic sensor works on the principle of SONAR(Sound Navigation and Ranging) i.e. sending a pulse of ultrasonic waves of frequency $44 \mathrm{kHz}$ when a signal of pulse width more than $10 \mathrm{~ms}$ is applied at the trigger input of the sensor. On occurrence of an obstacle, the ultrasonic waves get reflected and are sensed which in turn generate a pulse at the Echo pin of the ultrasonic sensor. The width of this pulse at the echo pin is measured in order to find the distance. The algorithm to develop android application is given below.

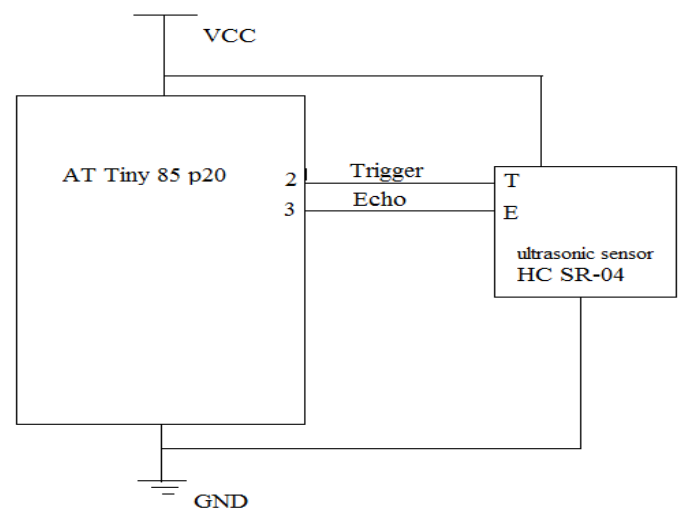

Fig. 2. Ultrasonic sensor interfacing

\section{Algorithm:}

1. Create Main Activity.

2. Create Check Availability Activity and download the updated availability file from the server and if available go to next screen.

3. Extract the available slots from the downloaded file and display on the screen.

4. Create Payment options Activity and select payment mode and go to next screen.

5. Create Payment details activity and accept details. 6. Generate OTP and send user's name and OTP to the server.

\section{RESULTS}

Some results of proposed systems are shown below to validate the proposed S-Park system.

\section{Development of Android Application:}

The Android Application needs to connect to the network, and then request server to check for the availability and thus download available spaces information from the server as shown in Figure 3.

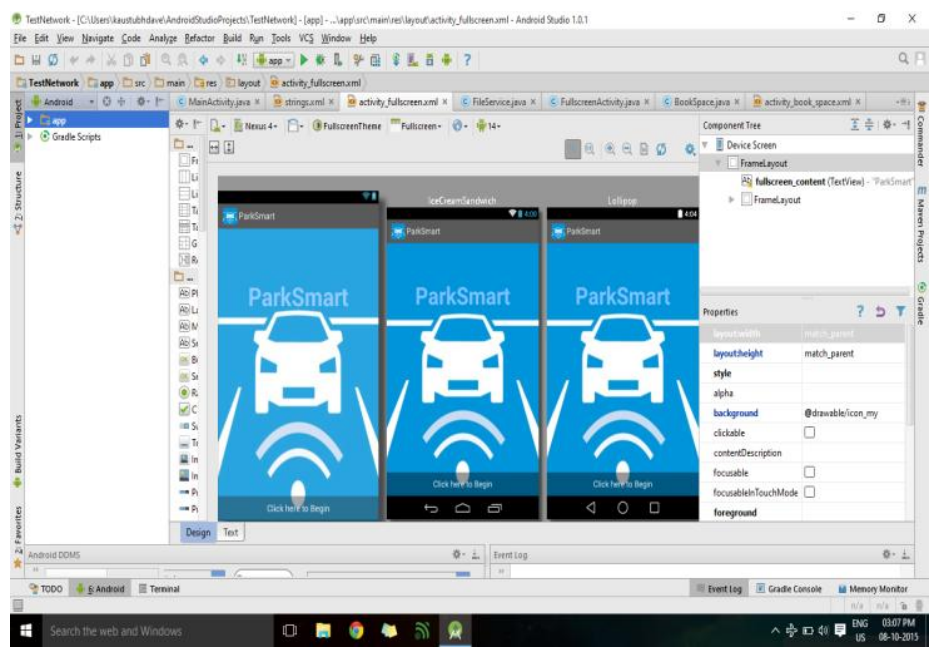

Fig. 3. Application screen test for various versions of android 
After opening this Android application, next step is checking availability of parking spaces on application as shown in Figure 4. The file is downloaded containing the current availability of the parking slots using HttpURLConnection. If the available spaces are zero then, the "Book Now" button is freezes as shown in Figure 5.

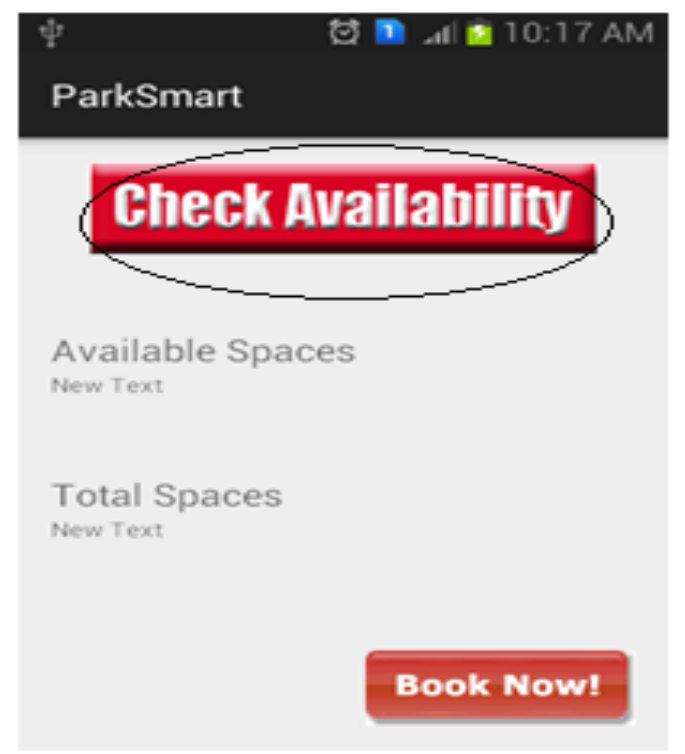

Fig. 4:Checking availability of parking spaces on application

Then on the BookSpace screen, the payment mode is selected by the user, and followed by "Pay Now" button as shown in Figure 6. User has to select either of the payment methods to continue. This is followed by Payment Activity wherein the user needs to enter the payment details which are then sent to the server as shown in Figure 7. The final screen in the application is the OTP generation screen where on successful payment an OTP (One Time Password) is generated which is sent to the server along with the user details. The user then exits the App using the "Exit" button as shown in Figure 8.

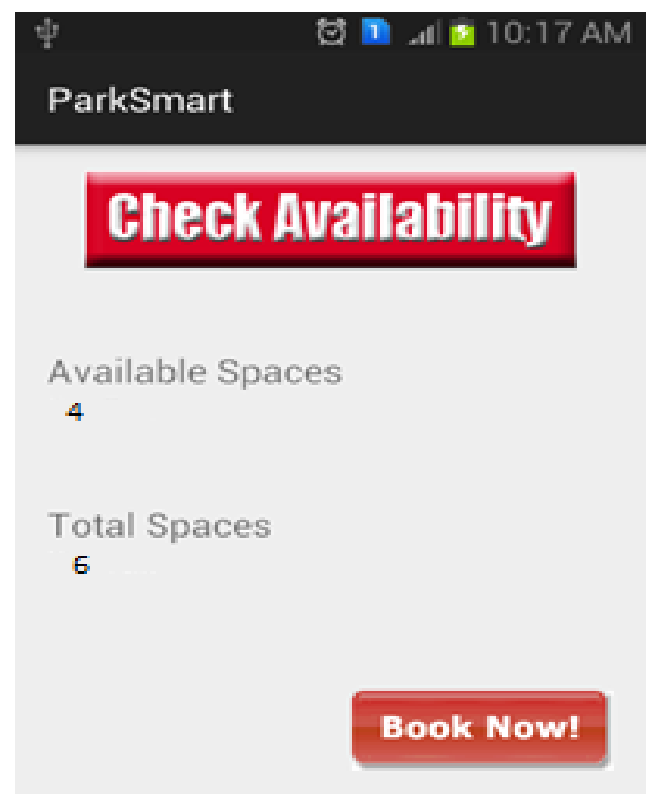

Fig.5:Checking availability of parking spaces on application

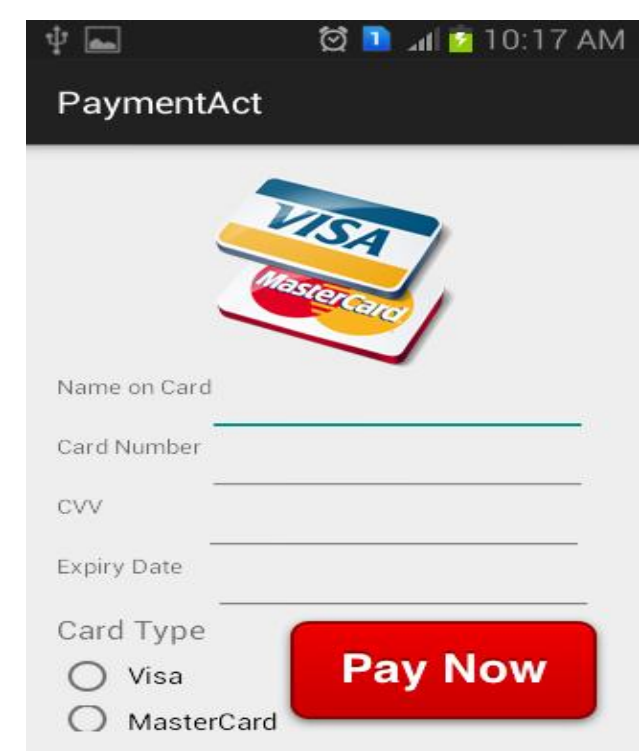

Fig. 6. Payment mode

The future work is to provide condition monitoring of electronics components used in system to improve performance without fail [6]-[11]. Also implement system based on solar energy as proposed in [12]-[13].

\section{CONCLUSION}

Finding a parking these days is a difficult task and generally results in traffic congestion and loss of time and fuel. This project aims at reducing such difficulties of the drivers by providing a reservation-based parking policy, thus alleviating their problems and making the process of finding a parking space easier. This project provides a solution for reservation based parking by keeping a time limit for the cars to arrive to reserved parking. This also avoids under utilization of the parking space. This project also implements the online reservation and payment facility so that there is assured parking available when driver reaches the parking. This system also provides online information of whether the parking if full or not. The Android app developed is user friendly as well as found to be working on different android phones with different screen resolutions. The app does not require any other features except internet connectivity to run. This system uses a wired network of sensors to connect to the embedded server this reduces overall cost of the system. It eliminates the need of additional hardware to be installed in cars. Ultrasonic sensors are used in this project to detect the cars in each slot this provides accurate as well as cost effective method to detect occupancy of the slot. The microcontroller based interface between server and sensors make the system reliable, cost effective, and extendible if number of slots needs to be increased after installation. Since number of cars is increasing at a fast rate but parking space does not increase to such an extent. There is need of such systems that require very less infrastructure for existing parking. 


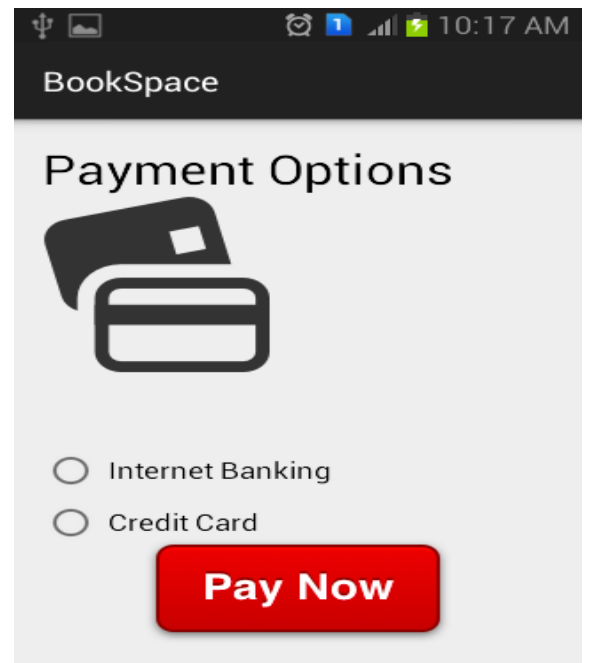

Fig. 7 : Functions of android application

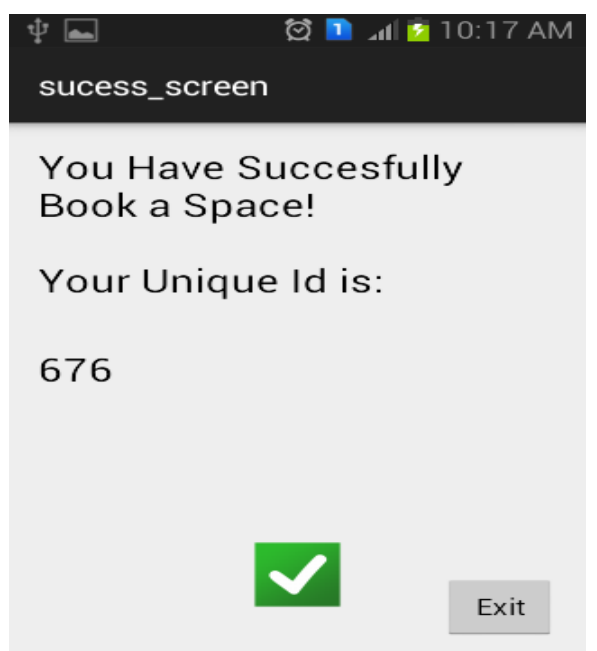

Fig. 8 : Functions of android application

\section{REFERENCES}

[1] Jihoon Yang, Jorge Portilla and Teresa Riesgo, "Smart Parking Service based on Wireless Sensor Networks", IEEE Magazine, Feb 2012, pp. 6029-6033.

[2] Yanfeng Geng, "New Smart Parking System Based on ResourceAllocation and Reservations", IEEE Transaction on intelligent transportation systems, Vol. 14, Issue No. 3, Sept 2013, pp. 1129-1138.

[3] Hongwei Wang and Wenbo He, "A Reservation-based Smart Parking System", The First International Workshop on Cyber-Physical Networking Systems, University of Nebraska-Lincoln, USA, Mar 2011, pp. 701-705.

[4] Behrouz Forouzan, "Data Communications and Networking", 4th edition, McGrawHill publications, India 2012, pp. 55-94.

[5] Willie D. Jones ,'Parking 2.0 meters go high tech", IEEE Spectrum, December 2006, pp. 20.

[6] R Khandebharad, R. B. Dhumale, S. S. Lokhande, "Electrolytic Capacitor Online Failure Detection and Life Prediction Methodology", Int. J. Research in Engg. And Techn., vol.4, Iss. 2, pp.636-641, Feb. 2015.

[7] R Khandebharad, R. B. Dhumale, S. S. Lokhande,
"Online Failure Prediction of the Aluminum Electrolytic Capacitor", Int. J. Science and Research, vol.4, Iss. 6, pp.2099-2102, June. 2015.

[8] R. B. Dhumale, S. D. Lokhande, N. D. Thombare, M. P. Ghatule, "Fault Detection and Diagnosis of High Speed Switching Devices in Power Converter", Int. J. Research in Engg. And Techn., vol.4, Iss. 2, pp.636641, Feb. 2015.

[9] M. R. Ubale, R. B. Dhumale, V. V. Dixit, S. D. Lokhande,“ Open Switch Fault Diagnosis Method in Three Phase Inverter", in Proc. National Conference on Latest Trends in Electronics and Telecommunication, Sinhgad College of engineering,Pune, Oct. 2013, pp28.

[10] M. R Ubale, R. B. Dhumale, S. D. Lokhande, "Method of Open Switch Fault Detection in Three Phase Inverter using Artificial Neural Network", Int. J. Research in Science and Advance Technology, Vol. 3, Iss. 3, PP 78-82, June 2014.

[11] Lalit Patil, R. B. Dhumale, S. D. Lokhande, "Fault Detection and Diagnosis Technique gor Power Inverter using Discrete Wavelet Transform", Int. J. Electronics Circuits and systems, Vol. 3, Iss. 2, pp 174-178, June 2014.

[12] C. Ugale , R. B. Dhumale , V. V. Dixit, “ DC-DC Converter for Solar Energy Harvesting using PID Controller", Int. J. Engineering Applications and Technology, Vol. 1, Iss. 13, pp 132-136, July 2105.

[13] C. Ugale , R. B. Dhumale , V. V. Dixit, "DC-DC Converter using Fuzzy Logic Controller", Int. J. Research in Engg. and Techn., vol.4, Iss.07, pp.490696, July. 2015. 